\title{
A proposed demonstration of an experiment of proton-driven plasma wakefield acceleration based on CERN SPS
}

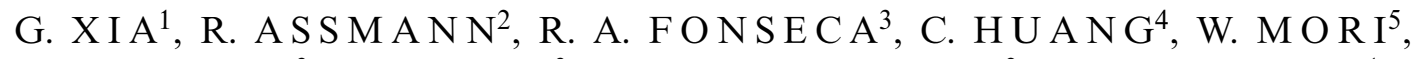 \\ L. O. S I L V A ${ }^{3}$, J. V I E I R A ${ }^{3}$, F. Z I M M E R A N N ${ }^{2}$ and P. M U G G I ${ }^{1}$ \\ for the PPWFA Collaboration \\ ${ }^{1}$ Max Planck Institute for Physics, Munich, Germany \\ (xiaguo@mpp.mpg.de) \\ ${ }^{2}$ CERN, Geneva, Switzerland \\ ${ }^{3}$ GoLP/Instituto de Plasmas e Fusao Nuclear-Laboratório Associado, IST, Lisboa, Portugal \\ ${ }^{4}$ Los Alamos National Laboratory, Los Alamos, NM, USA \\ ${ }^{5}$ University of California, Los Angeles, CA, USA
}

(Received 20 September 2011; accepted 2 January 2012; first published online 7 February 2012)

\begin{abstract}
The proton bunch-driven plasma wakefield acceleration (PWFA) has been proposed as an approach to accelerate an electron beam to the $\mathrm{TeV}$ energy regime in a single plasma section. An experimental program has been recently proposed to demonstrate the capability of proton-driven PWFA by using existing proton beams from the European Organization for Nuclear Research (CERN) accelerator complex. At present, a spare Super Proton Synchrotron (SPS) tunnel, having a length of $600 \mathrm{~m}$, could be used for this purpose. The layout of the experiment is introduced. Particlein-cell simulation results based on realistic SPS beam parameters are presented. Simulations show that working in a self-modulation regime, the wakefield driven by an SPS beam can accelerate an externally injected $\sim 10 \mathrm{MeV}$ electrons to $\sim 2 \mathrm{GeV}$ in a $10-\mathrm{m}$ plasma, with a plasma density of $7 \times 10^{14} \mathrm{~cm}^{-3}$.
\end{abstract}

\section{Introduction}

Over the past decade, there has been significant progress in developing the advanced accelerator concepts and possible technologies needed to produce and accelerate particle beams to high energies in short distances [1,2]. These so-called plasma accelerators have opened new research frontiers and hold great promise to decrease the scale and therefore the cost of future machines. Developments in these research areas have paved the way for a new generation of plasma accelerators not only for high-energy physics [3] but also for many other applications such as table-top x-ray Free Electron Lasers (FELs) [4], compact proton (carbon) treatment facilities, etc [5]. By employing an ultra-short, ultra-intense laser pulse as a driver, the Laser Wakefield Acceleration (LWFA) at Lawrence Berkeley National Laboratory (LBNL) has successfully demonstrated generation of $1 \mathrm{GeV}$ electron beam in a few centimeter long plasma cell [6]. With rapid progress in laser technology and laser guiding technique in plasmas, it is foreseen to reach a few tens of $\mathrm{GeV}$ electron acceleration for high-energy physics application [7]. Meanwhile, the electron beamdriven plasma wakefield acceleration (PWFA) experiment, conducted at the Final Focus Test Beam (FFTB) at SLAC, has doubled the energy of some fraction of electrons of the Stanford Linear Collider (SLC) beam [8] in only $85 \mathrm{~cm}$ of plasma, which corresponds the wakefield amplitude in excess of $50 \mathrm{GeV} / \mathrm{m}$, more than two orders of magnitude higher than the current radiofrequency technology in use. The next generation PWFA experiment foreseen at FACET will accelerate a separate witness electron bunch to high energy with narrow energy spread and preserved emittance [9]. However, to reach the energy frontier of Teraelectron volts (or $\mathrm{TeV}$ ) energy regime, both LWFA and PWFA still face some practical challenges, for instance, synchronizing and aligning of many similar accelerating modules may become difficult.

In general, plasma acts as an energy transformer in both LWFA and PWFA. The plasma itself cannot produce the net energy. However, it is a perfect medium to transfer energy from a driver pulse (beam) to the witness beam. Since plasma is already an ionized (broken down) medium, there is no further breakdown. It can therefore sustain very large electric fields. In contrast, the metallic radiofrequency (RF) structures, from which the particles gain energies in conventional accelerators, are subject to material breakdown in high electric field. Generally, the conventional RF cavity cannot endure the electric field higher than $200 \mathrm{MeV} / \mathrm{m}$ [10].

More recently, Caldwell et al. [11] proposed a new scheme, the so-called 'proton-driven plasma wakefield acceleration'. The underlying physics is similar to the other beam-driven plasma wakefield acceleration. That is, the space charge of the drive beam sets the ambient plasma electrons in motion. The heavy plasma ions 
are stationary and therefore provide restoring forces to plasma electrons and make them bounce in and out from the beam propagation axis. The very quick movement of plasma electrons corresponds to a very high-frequency electric field. Sitting in the right phase, some particles, either on the rear part of the drive beam or an externally injected witness beam will gain energies from this wakefield (wake). For a longitudinally symmetric bunch, the transformer ratio (ratio of electric field seen by the witness particles to that seen by the drive particles) is limited to 2 [12]. That is to say, when the drive and witness beams start with the same energy, the drive beam can lose all of its energy, and in return the witness beam could at most triple its energy. Employing the present most energetic electron beam from the SLC as a drive beam, the maximum energy that can be achieved through PWFA is around $150 \mathrm{GeV}$ in a single stage of acceleration. This energy is lower than what the high-energy physics community requires, and hence staging is required. Taken this into consideration, using $\mathrm{TeV}$ protons as the drive beam, it should be possible to reach the $\mathrm{TeV}$ scale energy gain for the injected electron beam. The advantage of using a proton beam as a driver is that nowadays there are a few $\mathrm{TeV}$ proton synchrotron facilities around the world, e.g. Tevatron, HERA, and the LHC. The energy stored in a typical $\mathrm{TeV}$ scale proton bunch $(\sim 100 \mathrm{~kJ})$ is two or three orders magnitude higher than that of the highest energy electron beam from $\operatorname{SLC}(\sim 100 \mathrm{~J})$. The idea behind this proton-driven plasma wakefield acceleration scheme is to transfer energy from highenergy protons to the plasma and then to the electrons. In this scenario, a proton beam is sent into a pre-formed plasma. Particle-in-cell (PIC) simulations show that a $1 \mathrm{TeV}$ proton bunch with a drive bunch population of $10^{11}$ and a bunch length of $100 \mu \mathrm{m}$ injected in a preionized uniform plasma with density of $6 \times 10^{14} \mathrm{~cm}^{-3}$ excites a wakefield with an amplitude of $\sim 2 \mathrm{GeV} / \mathrm{m}$. An externally injected $10 \mathrm{GeV}$ electron bunch witnesses this accelerating field and reaches a final energy greater than $600 \mathrm{GeV}$ in a single plasma section of $450 \mathrm{~m}$ [11]. If this could be demonstrated, it would potentially open a new research frontier to accelerate electron beams to $\mathrm{TeV}$ energy regime (energy frontier). This exciting simulation result has aroused great interests in the community. A wide collaboration has been formed to investigate the underlying physics and the key issues in realizing an experimental test of the proton-driven plasma wakefield acceleration [13].

\section{Self-modulation of a long beam}

In the plasma wakefield acceleration, an efficient excitation of wakefield requires the bunch length $\sigma_{z}$ close to the plasma wavelength $\lambda_{p}=2 \pi c / \omega_{p}$, where $c$ is the speed of light. $\omega_{p}=\sqrt{n_{p} e^{2} / \varepsilon_{0} m}$ is the plasma frequency, $n_{p}$ is the plasma density, $e, m$, and $\varepsilon_{0}$ are electron charge, electron mass, and the permittivity of free space, respectively. However, for the proton-driven plasma wakefield acceleration, the available high-energy beams from current proton synchrotrons are quite long. For example, the proton bunch length for Tevatron, HERA, and LHC are 50, 8.5 and $7.55 \mathrm{~cm}$, respectively, which is much longer than the plasma wavelength of interest for high-gradient acceleration. The proton bunches are long so as to avoid beam instabilities in the ring. For example, in the European Organization for Nuclear Research (CERN) accelerator complex, the longitudinal emittance is blown up intentionally by noise injection in order to create the Landau damping against instabilities. Meanwhile, another limiting factor on the bunch length is the beam current (that scales as the charge divided by the bunch length), which gives some constraints due to transverse single- and multi-bunch instabilities.

Compression of high-energy proton bunches to submillimeter length, the conventional magnetic chicane seems very challenging, since it requires large amounts of $\mathrm{RF}$ powers to introduce the necessary energy chirp along the beam and large dipoles to create a dispersive section for path modulation. Preliminary simulation studies show that to compress a $1-\mathrm{TeV}, 1-\mathrm{cm}$ proton bunch to $\sim 100 \mu \mathrm{m}$ requires a $4-\mathrm{km}$ linac to provide enough energy chirp. It is therefore too expensive and impractical [13]. However, when a beam with a bunch length much longer than the plasma wavelength is injected into a highdensity plasma, the particles in the bunch head will excite wakefields. The transverse wakefield will modulate the bunch current. Subject to the density modulation effect, the long proton beam will be split into many short bunch slices due to the transverse focusing and defocusing field [14]. After propagating some distances, a full selfmodulation will be formed. These bunch slices will coherently excite the wakefield and eventually the fields add up to a higher amplitude. PIC simulation shows that working in self-modulation regime, the wakefield amplitude (on-axis electric field) can reach from several hundred $\mathrm{MeV} / \mathrm{m}$ up to $1 \mathrm{GeV} / \mathrm{m}$ by employing the available proton beam from Super Proton Synchrotron (SPS) at CERN. This process very much resembles the self-modulated laser wakefield acceleration scheme (SMLWFA), in which a long laser pulse (compared with the plasma wavelength) is modulated by the wakefield and in turn a long laser pulse is split into many short pulses. Therefore a high-density plasma can be used to excite large amplitude wakefields even with long laser pulses $[15,16]$.

\section{SPS beam for the experiment}

In order to demonstrate the capability of the protondriven plasma wakefield acceleration, we proposed an experimental test of this scheme based on the CERN accelerator complex. Fortunately, there is currently a spare tunnel from the SPS, with a footprint of $620 \mathrm{~m}$ in the West Area, which could be used for the proposed experimental program. As an injector, the SPS can 
Table 1. Parameters for SPS-LHC and SPS-optimum beams.

\begin{tabular}{lll}
\hline & SPS-LHC & SPS-optimum \\
\hline Beam energy $(\mathrm{GeV})$ & 450 & 450 \\
Bunch population $\left(10^{11}\right)$ & 1.15 & 3.0 \\
Beam radius $(\mu \mathrm{m})$ & 200 & 200 \\
Angular spread $(\mathrm{mrad})$ & 0.04 & 0.04 \\
Normalized emittance $(\mu \mathrm{m})$ & 3.5 & 3.5 \\
Bunch length $(\mathrm{cm})$ & 12 & 12.4 \\
Energy spread $(\%)$ & 0.03 & 0.03 \\
\hline
\end{tabular}

provide very intense and high-energy proton beams for the LHC and other experiments such as CNGS and HiRadMat. The maximum beam energy from SPS is $450 \mathrm{GeV}$, with a nominal bunch population of $1.15 \times 10^{11}$ and an rms bunch length of $12 \mathrm{~cm}$. The transverse normalized emittance is about $3.5 \mathrm{~mm} \cdot \mathrm{mrad}$ and the relative energy spread is $3 \times 10^{-4}$. The SPS-LHC beam parameters are listed in Table 1.

Based on the linear theory of PWFA, a short drive beam with a bunch length fraction of the plasma wavelength is needed to resonantly excite a large amplitude wakefield. However, compression of SPS beam from initially $12 \mathrm{~cm}$ to hundred microns approximately (the same scale as the plasma wavelength at a density of around $10^{15} \mathrm{~cm}^{-3}$ ) seems difficult [17]. In order to keep the cost of the first experiment as modest as possible, the beam will not be compressed, but rather a long proton beam will be directly sent into the plasma and we will study interactions between a long proton bunch and the plasma. Since the proton bunch is much longer than the plasma wavelength, we expect that a strong self-modulation will occur within the body of beam due to transverse instability. Subject to this modulation effect, the long proton bunch splits into many ultra short beam slices, with periodicity of a plasma wavelength [14]. Figure 1 shows the on-axis SPS beam density profile $(X=0)$ after 5 -m propagation in plasma with a density of $10^{14} \mathrm{~cm}^{-3}$. The proton bunch density is clearly modulated by the plasma wakefield. The length of each bunch is around one-half of the plasma wavelength. These ultra-short bunches resonantly excite the plasma wakefield, and the wakefield can potentially be used to accelerate both some of the protons and externally injected electrons.

In the first experiment, we anticipate demonstrating the acceleration of an externally injected $\sim 10 \mathrm{MeV}$ electron beam to $1 \mathrm{GeV}$ with an SPS proton bunch as driver in a $5-10-\mathrm{m}$ plasma. Based on the success of first round of experiments, we will further study acceleration of an electron bunch to $100 \mathrm{GeV}$ in a $100-\mathrm{m}$ plasma. The schematic of the beam line layout for our demonstration experiment is shown in Fig. 2. After being switched into the proposed experimental tunnel TT61, the SPSextracted beam will follow a 400-m transfer line with a slope of $6-7 \%$ (in the future experiment, this tunnel may also be used for beam manipulation, e.g. bunch length and density shaping) and finally reach the ground surface areas TT4 and TT5. The beam properties, such as transverse size, beam angular spread, etc., will also be adjusted to match plasma parameters. We assume that a 5-10-m-long homogenous plasma is produced either by a laser-ionized metal vapor [18], a helicon plasma source [19], or a discharge plasma source. To witness the wakefield, we will inject a long electron beam (in future, we may also employ a compact laser plasma injector to produce high quality electrons with ultra-short bunch length), with an energy of tens of $\mathrm{MeV}$ (relativistic regime) before a plasma cell. Electrons injected at a right phase will reach high energies, while others will be decelerated. Exiting the plasma cell, an energy spectrometer will be used to analyze the electron beam energy variation after the plasma. Diagnostics will be used to characterize beam properties (beam size, current, emittance, self-modulation effect, electron/proton energy variation, etc.) with and without the plasma present. The spent proton beam will be absorbed in the beam dump area.

\section{Wakefield driven by SPS beam}

The main parameters of a nominal SPS beam (or a standard SPS-LHC beam for injection into the LHC) and an optimum SPS beam (or SPS-optimum beam, a high-density beam that can be produced in a single bunch operation mode in SPS) are listed in Table 1. The beam density for an optimum SPS is $\sim 2.5$ times higher than that of the SPS-LHC beam. It is expected that the optimum SPS beam can therefore initially excite a larger electric field in the plasma. Various PIC and hybrid codes have been used to simulate interactions between the SPS beams and the plasmas. Most results have already been compared and benchmarked and found to be in good agreement with each other. Figure 3

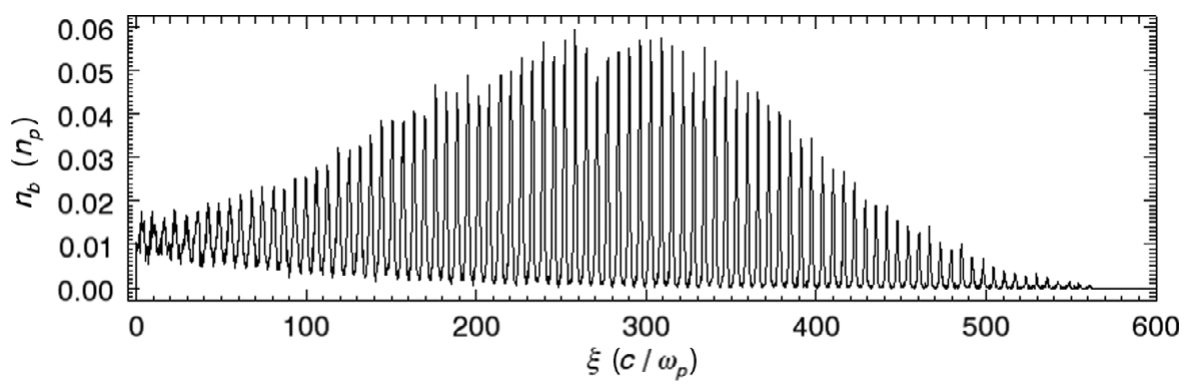

Figure 1. On-axis $(X=0)$ beam density profile after 5-m propagation in plasma. 


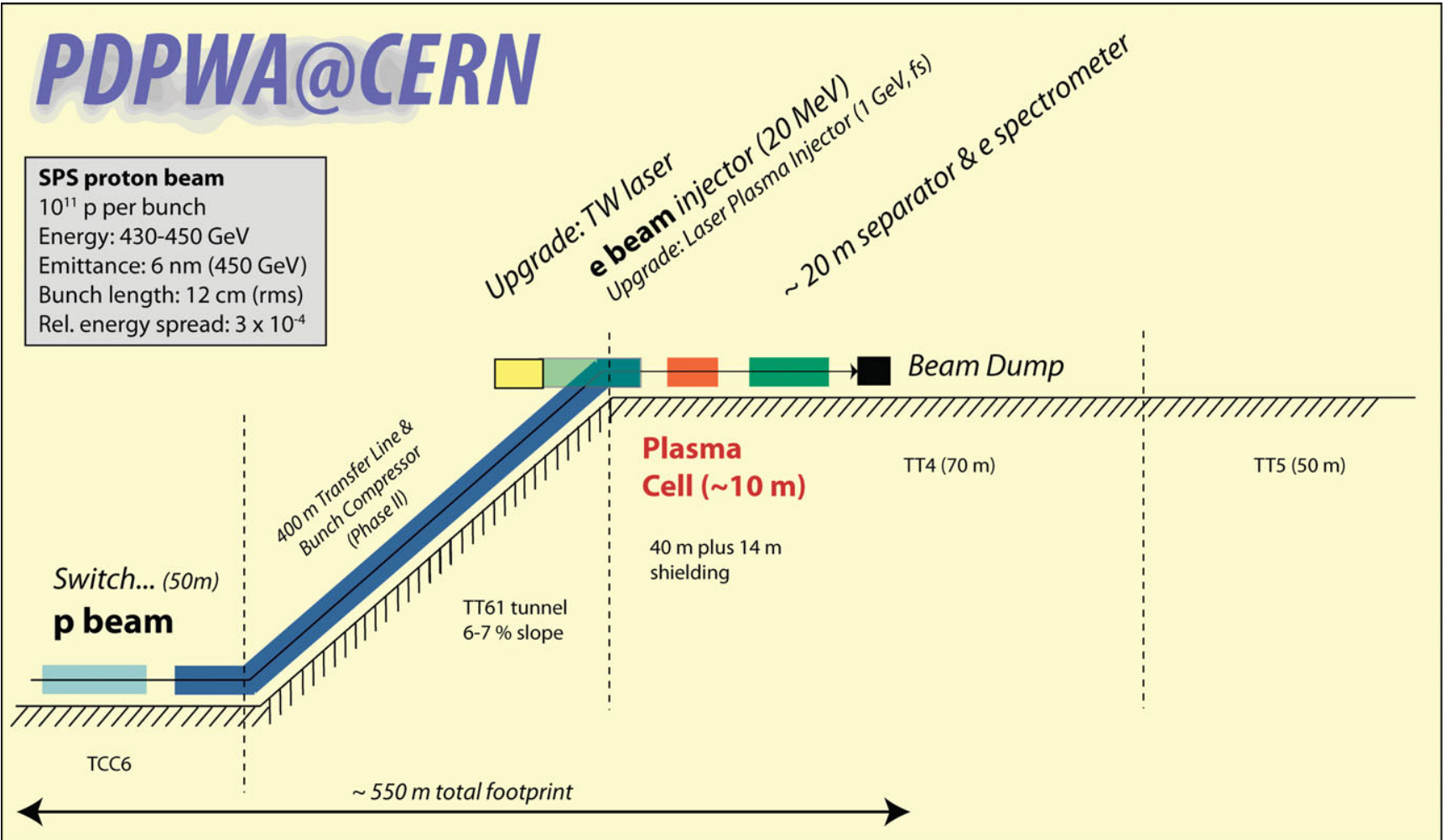

Figure 2. (Colour online) Schematic of a beam-line layout for proton-driven PWA experiment at CERN.

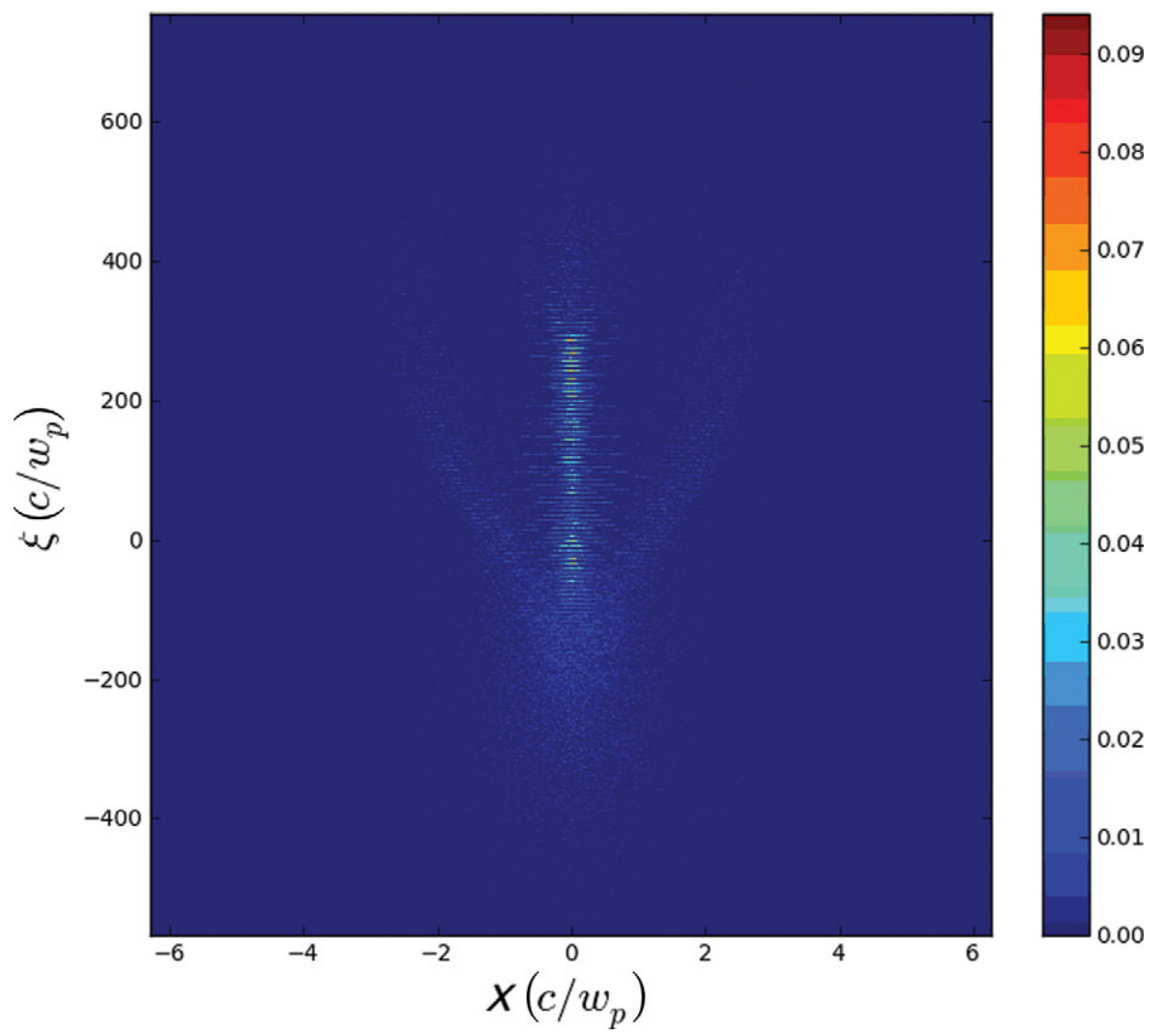

Figure 3. (Colour online) Beam density distribution at 10-m plasma, as obtained from the numerical code QuickPIC [20]. The proton bunch propagates from top to bottom. 


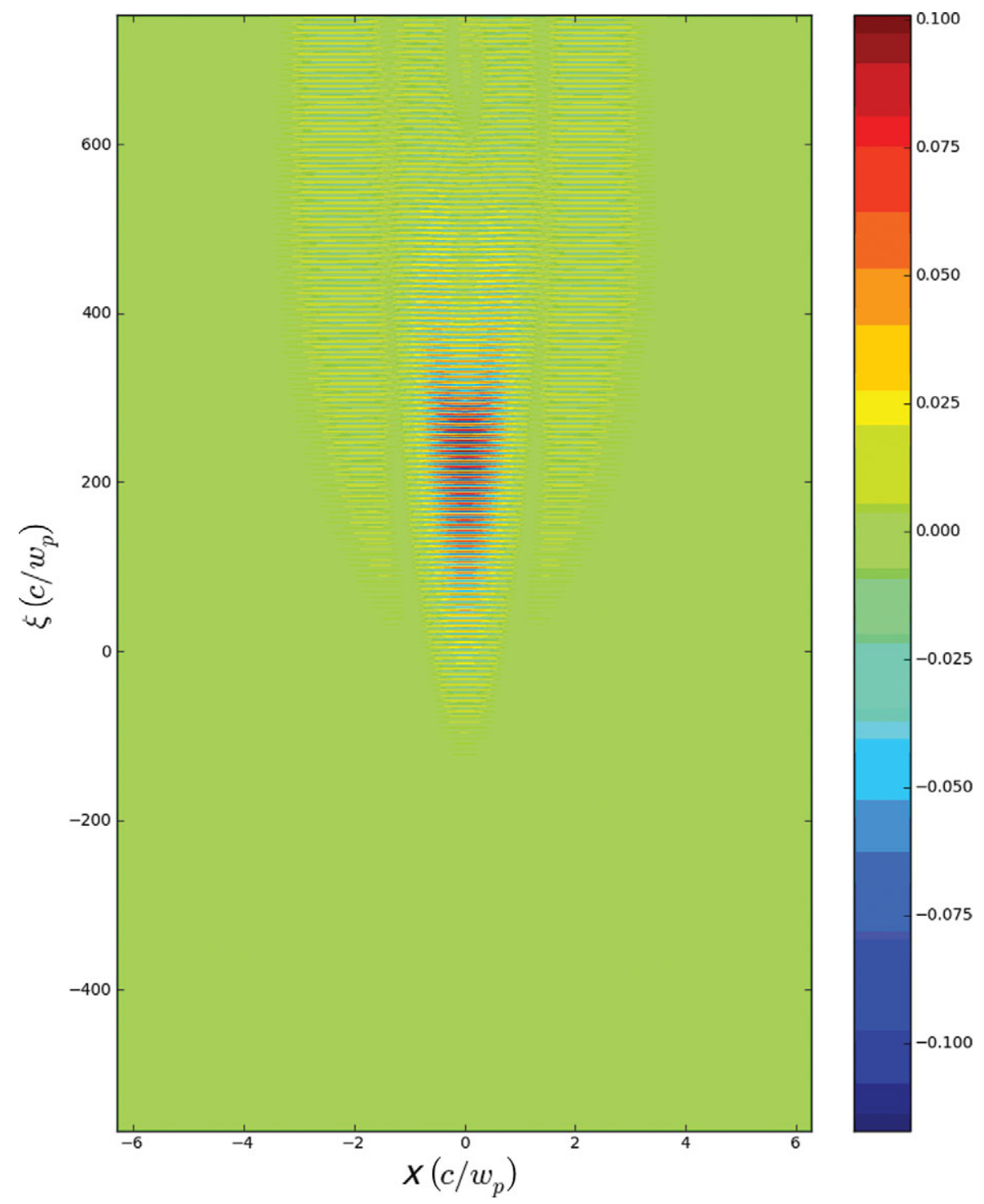

Figure 4. (Colour online) Longitudinal electric field at 10-m plasma, as obtained from the numerical code QuickPIC [20]. The proton bunch propagates from top to bottom.

shows an uncompressed SPS-LHC beam density distribution (normalized to plasma density in the right color bar) after $10-\mathrm{m}$ plasma with a density of $10^{14} \mathrm{~cm}^{-3}$. It was obtained using the quasi-static particle-in-cell code QuickPIC [20]). Here $\xi(\xi=c t-z)$ denotes the distance in the beam frame (beam propagating downwards along $\xi$ axis) with $\xi=0$ in the middle of the bunch and $X$ denotes the transverse horizontal direction. It is clearly seen that the beam density is fully modulated after $10-\mathrm{m}$ plasma (with a maximum beam density $=\sim 0.09 n_{\mathrm{p}}$, note that the ratio between the initial SPS-LHC beam density and the plasma density is $0.015 n_{\mathrm{p}}$ ). Some particles in the bunch (in the focusing phase of the wake) are focused and the beam density becomes denser locally. Other particles (in the defocusing phase of the wake) are defocused and scattered transversely leading to a large transverse size and lower beam density regions. Since it takes some time for the self-modulation effect to develop, we found that protons in the bunch tail reach very large modulation, while some fraction of protons in the bunch head are scattered transversely due to less focusing field from the plasma wakefield. This modulated bunch pattern can propagate for a long distance. Figure 4 shows the longitudinal electric field (normalized to the wave breaking field, $E_{w b} \approx 100 \sqrt{n_{p}}(V / m)$, here $n_{\mathrm{p}}$ is in units of $\mathrm{cm}^{-3}$ ) excited by an uncompressed SPS-LHC beam after $10 \mathrm{~m}$ of plasma. It shows that the field amplitude of $\sim 100 \mathrm{MeV} / \mathrm{m}$ can be achieved with a relatively low plasma density of $10^{14} \mathrm{~cm}^{-3}$.

More recently, we performed 2D cylindrically symmetric OSIRIS simulations [21] and compared the wakefield amplitude driven by a half-cut SPS-LHC beam and a half-cut SPS-optimum beam. The purpose of using a half-cut beam is to seed instability. In this case, the plasma will see the proton bunch with a very sharp current flank. The instability seeded by this flank quickly modulates the density of the proton bunch. Figure 5 shows that with a plasma density of $7 \times 10^{14} \mathrm{~cm}^{-3}$, the 


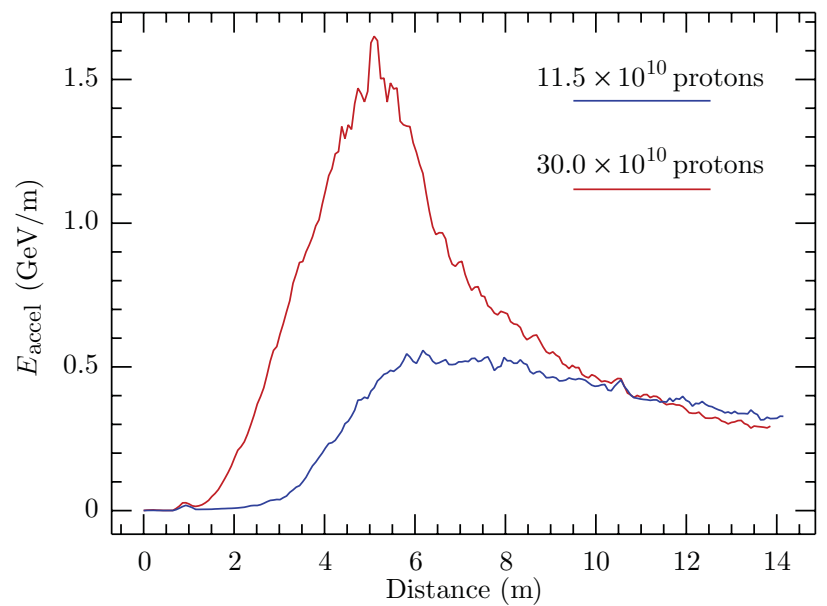

Figure 5. (Colour online) The maximum longitudinal electric fields for SPS-LHC and SPS-optimum beams with respect to the travelled distance.

maximum longitudinal electric field driven by a halfcut SPS-LHC beam is beyond $500 \mathrm{MeV} / \mathrm{m}$ after $6 \mathrm{~m}$. For the SPS-optimum beam, the wakefield amplitude is over $1.5 \mathrm{GeV} / \mathrm{m}$ after $5 \mathrm{~m}$ in the plasma. Compared with Fig. 4, we found that a higher amplitude wakefield can be achieved at higher plasma density. However, the proton beam is subject to a severe hosing instability at high plasma density and a compromise will be worked out to choose an optimum plasma density for stable high-gradient acceleration.

\section{Simulation of electron injection}

Based upon PIC simulation results, the energy change of the SPS proton beam in a $10-\mathrm{m}$ plasma (with a plasma density of $10^{14} \mathrm{~cm}^{-3}$ ) is around $1-2 \mathrm{GeV}$, which is not significant compared with the initial proton beam energy, and therefore it is difficult to be observed in the experiment. We therefore plan to inject an external electron beam for the diagnostic of the wakefield (as shown in Fig. 2). We have performed a $2 \mathrm{D}$ cylindrically symmetric simulation using OSIRIS to check the electron acceleration process in the wakefield driven by a self-modulated proton bunch. Figure 6 shows the maximum electron energy gain as a function of the distance travelled in the plasma. The injected electron beam energy is $10 \mathrm{MeV}$, which is in the relativistic regime. The other electron beam parameters are as follows: number of particles: $10^{6}$, rms length: $100 \mathrm{~mm}$, rms transverse normalized emittance: $2 \mathrm{~mm} \cdot \mathrm{mrad}$, beam size: $200 \mu \mathrm{m}$, and the divergence angle: $0.1 \mathrm{mrad}$. The electron beam co-propagates with the proton beam and some electrons will gain energy from the wakefields. The simulation shows that after the $10-\mathrm{m}$ plasma (with a density of $7 \times 10^{14} \mathrm{~cm}^{-3}$ ), some electrons have gained energies greater than $0.5 \mathrm{GeV}$ (blue curve) with the nominal SPS-LHC beam as a drive beam. For the SPSoptimum beam, the maximum energy gain is beyond $2.0 \mathrm{GeV}$ in a $10-\mathrm{m}$ plasma (red curve). Figures 7 and

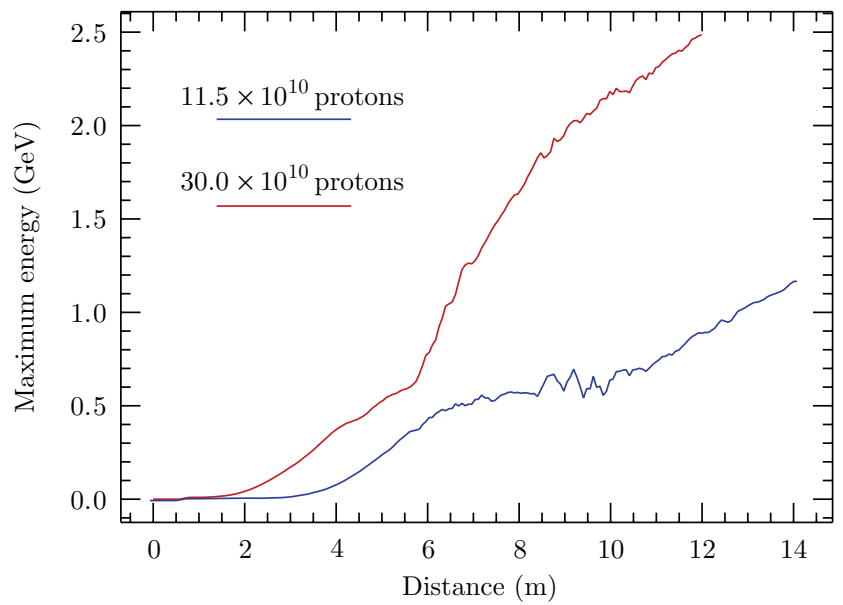

Figure 6. (Colour online) Maximum energy of externally injected electrons as a function of the travelled distance in plasma.

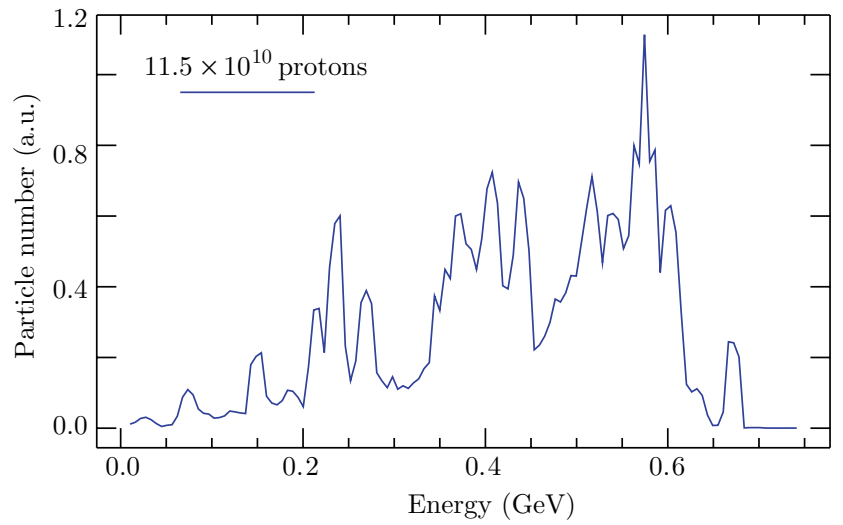

Figure 7. (Colour online) Spectrum of an externally injected electron beam after $10 \mathrm{~m}$ of propagation using nominal SPS-LHC beam as driver.

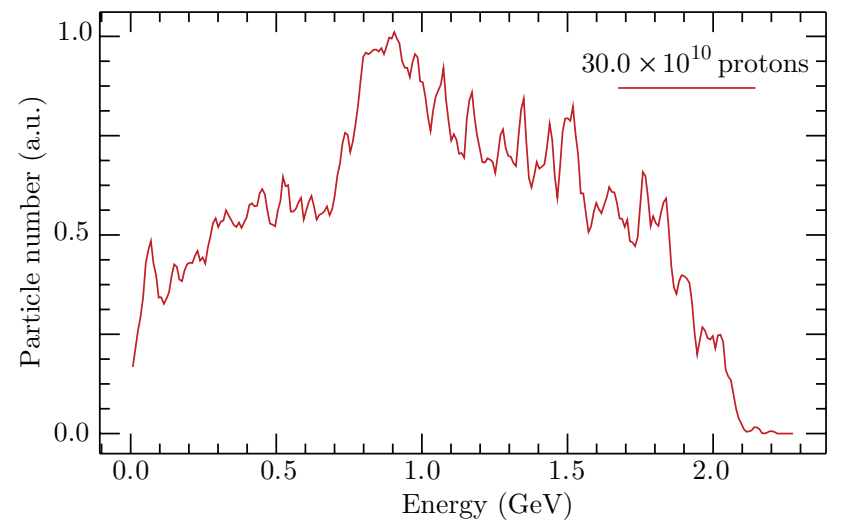

Figure 8. (Colour online) Spectrum of an externally injected electron beam after $10 \mathrm{~m}$ of propagation using SPS-optimum beam as driver.

8 give the energy spectra of injected electrons after $10-\mathrm{m}$ propagation in the plasma with a density of $7 \times 10^{14} \mathrm{~cm}^{-3}$ for SPS-LHC beam and SPS-optimum beam, respectively. It can be seen that the electron beam has a wide energy spread in both cases. For the SPS-optimum beam, the maximum electron energy reaches up to $2 \mathrm{GeV}$. We expect that a larger 
energy gain and a narrow energy spread beam can be achieved by optimizing the electron beam and plasma parameters (beam energy, bunch charge, bunch length, emittance, plasma density, etc.) and by controlling the injection position of a short electron bunch with respect to the phase of the wakefields [22]. This optimization work is still underway and will be reported elsewhere.

\section{Conclusion}

We presented a proposed experimental study of a proton-driven plasma wakefield accelerator. By sending a long proton bunch into a homogeneous plasma, selfmodulation occurs due to transverse instability resulting from the transverse plasma wakefields. The resulting many ultra-short bunches excite the wakefield coherently and the field amplitude grows. For our proposed proton-driven plasma wakefield acceleration experiment using the SPS beam as the driver, PIC simulations show that an accelerating gradient of several hundred $\mathrm{MeV} / \mathrm{m}$ can be achieved. For the SPSoptimum beam parameters, the wakefields amplitude is much higher. An externally injected relativistic electron beam $(\sim 10 \mathrm{MeV})$ can sample the wakefield and gain energies up to $1 \sim 2 \mathrm{GeV}$ in a 10 -m-long plasma cell.

\section{References}

[1] Esarey, E. et al. 2009 Rev. Mod. Phys. 81, 1229.

[2] Joshi, C. and Malka, V. 2010 New J. Phys. 12, 045003.

[3] Muggli, P. and Hogan, M. J. 2009 C. R. Phys. 10, 116.

[4] Nakajima, K. et al. 1999 Nucl. Instrum. Meth. A 375, 593.

[5] Tajima, T. et al. 2009 Rev. Accel. Sci. Technol. 2, 201.

[6] Leemans, W. P. et al. 2006 Nature Phys. 2, 696.

[7] Leemans, W. P. et al. 2011 Proc. Particle Accelerator Conference, New York, USA, March 27-April 1.

[8] Blumenfeld, I. et al. 2007 Nature 445, 741.

[9] Hogan, M. J. et al. 2010 New J. Phys. 12, 055030.

[10] Braun, H. H. et al. 2003 Phys. Rev. Lett. 90, 224801.

[11] Caldwell, A. et al. 2009 Nature Phys. 5, 363.

[12] Ruth, R. et al. 1985 Part. Accel. 17, 171.

[13] Xia, G. et al. 2010 Advanced accelerator concepts. Proc. AIP Conf. 1299, 510.

[14] Kumar, N. et al. 2010 Phys. Rev. Lett. 104, 255003.

[15] Esarey, E. et al. 1994 Phys. Rev. Lett. 72, 2887.

[16] Mori, W. B. 1997 IEEE J. Quantum Electron. 33, 1942.

[17] Xia, G. and Caldwell, A. 2010 Proc. IPAC'10 Kyoto, Japan, p. 4395.

[18] Muggli, P. et al. 1999 IEEE Trans. Plasma Sci. 27, 791.

[19] Arnush, D. and Chen, F. F. 1998 Phys. Plasmas 5, 1239.

[20] Huang, C. et al. 2006 J. Comp. Phys. 217, 658.

[21] Fonseca, R. A. et al. 2002 Lect. Notes Comp. Sci. 2331, 342.

[22] Lotov, K. V. 2011 Phys. Plasmas 18, 024501. 